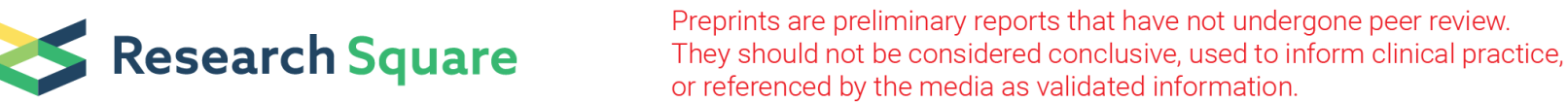

\section{Tomotherapy for Advanced Cervical Cancer: A Comparison of Dosimetric and Clinical Outcomes With Intensity Modulated Radiation Therapy}

\section{Yueju Yin}

Shandong Tumor Hospital and Institute

Dandan Wang

Shandong Tumor Hospital and Institute

Dapeng Li ( $\nabla$ drldp@126.com)

Department of Gynecologic Oncology, Shandong Cancer Hospital and Institute, Shandong First Medical University and Shandong Academy of Medical Sciences, Jinan 250117, China. https://orcid.org/00000002-0483-2803

Jian Zhu

Shandong Tumor Hospital and Institute

\section{Quancai Chen}

wulian matenal and child care service center

\section{Xiugui Sheng}

national clinical research center of cnacar

\section{Shuai Feng}

Shandong Tumor Hospital and Institute

Jue Jia

Shandong Tumor Hospital and Institute

Hao Yu

Shandong Tumor Hospital and Institute

\section{Research}

Keywords: Intensity-modulated radiation therapy (IMRT), tomotherapy (TOMO), leukopenia

Posted Date: August 31st, 2020

DOI: https://doi.org/10.21203/rs.3.rs-59610/v1

License: (1) (1) This work is licensed under a Creative Commons Attribution 4.0 International License.

Read Full License 


\section{Abstract}

Purpose: The aim of this study was to investigate the therapeutic response (loco-regional control, diseasefree, overall survival), toxicities (acute and long-term toxicities) and dosimetric parameters between intensity-modulated radiation therapy (IMRT) and tomotherapy (TOMO) in patients with advanced cervical cancer.

Materials and Methods: This study included 310 patients with advanced cervical cancer and who received concurrent chemoradiation (CCRT) from August 2015 to March 2018. All patients were initially diagnosed with International Federation of Gynecology and Obstetrics (FIGO 2009) stage $\triangle \mathrm{B}-\triangle \mathrm{B}$ cervical cancer. A total of 155 patients were treated with TOMO, whereas the remaining 155 patients underwent IMRT. Intracavitary brachytherapy and concurrent chemotherapy were performed during external irradiation.

Results: A total of 310 patients were included in the present study. There was no statistical difference in age, FIGO stage, histologictype, tumor size, tumor grade, pathologic morphology type, between the 2 groups ( $P=0.924,0.352,0.954,0.36,0.11$, and 0.123 , respectively). In comparing TOMO with IMRT, better dose conformity $(0.82 \pm 0.033$ vs. $0.75 \pm 0.064, P=0.006)$ and dose homogeneity $(1.03 \pm 0.006$ vs. $1.09 \pm 0.076, \mathrm{p} \otimes 0.001)$ were observed by TOMO planning. TOMO provided better critical organ sparing than IMRT in the lower bladder V20 ( $p=0.001)$, femoral head V40 $(p=0.014)$, and lower rectum V40 $(p=0.035)$, V20 $(p=0.005)$ were observed in the planning by TOMO compared to IMRT for patients with advanced cervical cancer. TOMO demonstrated a superior ability to protect the ovary (Dmax: 4.61Gy versus 5.81Gy, $P=0.026$; Dmean: $2.99 \mathrm{~Gy}$ versus $3.97 \mathrm{~Gy}, \mathrm{P}=0.017$ ). A few OARs and dosimetric parameters, including bladder V40 $(p=0.113)$, and femoral head V20 $(p=0.066)$, exhibited a tendency toward more favorable values in TOMO than IMRT. There were no statistically signifcant diferences in small bowel V20 $(p=0.251), V 40(p=0.575)$ or bone marrow protection V20 $(p=0.917), V 40(p=0.53)$ between the IMRT plan and the TOMO plan. But it gave significantly better values for Dmax parameters for bone marrow and small bowel with a statistically significant level ( $P=0.004$, and 0.002 , respectively). Acute major toxic effects included cystitis, proctitis, leukopenia, dermatitis, and enteritis. Seventeen (11\%) patients in the IMRT group and 5(3.22\%) in the TOMO group experienced grade 3/4 acute proctitis. Grade 3/4 leukopenia occurred in 71 (45.81\%) patients in the IMRT group and 60 (38.71\%) patients in TOMO group. Eleven $(7.1 \%)$ patients in the IMRT group experienced grade $3 / 4$ late radiation cystitis. And grade $3 / 4$ late radiation enterocolitis occurred in $10(6.45 \%)$ patients in the IMRT group. The incidence of chronic radiation cystitis and enterocolitis in the TOMO group was 3.87\% (6/155). The acute radiation toxicity of proctitis, and leukopenia was significantly lower in TOMO group than IMRT group $(P=0.034$, and 0.025 , respectively). There was no statistical difference in the acute radiation toxicity of cystitis, enteritis, and dermatitis between the 2 groups ( $P=0.084,0.082$ and 0.616 , respectively). The chronic radiation toxicity of radiation enterocolitis and cystitis was lower in the IMRT group ( $P=0.032$ and 0.048 , respectively). But there was no statistical difference for 1- and 3-year OS between the TOMO and IMRT groups $(98.7 \%$ vs $98.5 \%, P=0.149 \llbracket 91.3 \%$ vs $96.3 \%, P=0.142$ ). No obvious difference was found in 1 - and3-year $P F S$ rates between 2 groups (1-year: $91.4 \%$ vs $91.6 \%, P=0.82$; 3 -year: $86.8 \%$ vs $88.3 \%, P=0.751$ ). 
Conclusions: This study has shown that TOMO and IMRT are comparable in dose conformity, dose homogeneity, and protection of the ovary. TOMO provided better critical organ sparing than IMRT in lower bladder, femoral head, ovary sparing and lower rectum were observed in the planning. The acute and chronic toxicities were acceptable. So TOMO is a good option for adjuvant treatment of FIGO stage $\triangle \mathrm{B}-$ $\triangle \mathrm{B}$ cervical cancer, especially to young patients. Further prospective randomized multicenter studies are needed to confirm the benefits of TOMO.

\section{Introduction}

Cervical cancer is a disease that affects millions of women worldwide; it ranks as the fourth most frequent cancer in women globally [1]. The incidence of cervical cancer is on the rise in China. It caused approximately 98,900 new cases and 30,000 deaths in 2015[2]. A combination of external beam radiation therapy (EBRT) and brachytherapy (BT) with concurrent chemotherapy is the standard treatment of locally advanced cervical cancer. Over the last two decades, development of sophisticated planning and delivery techniques, and introduction of computer technology and imaging have galvanized the practice of radiotherapy, resulting in improved clinical outcome and reduced toxicity [3.4].

The delivery of adequate radiation dose to the cervical tumor area through the traditional approach is limited by the normal structures in the pelvic cavity that are sensitive to radiation, including the bladder and rectum. Helical tomotherapy (TOMO) is a novel radiation therapy modality [5]. It is a form of intensity-modulated radiation therapy (IMRT) that uses a helical $360^{\circ}$ radiation delivery system. It delivers image-guided radiation therapy through comparison of daily pretreatment megavoltage CT (MVCT) scans with CT scans performed at the time of simulation for treatment planning. By rapid opening and closing of leaves in a collimator rotating around the patient, TOMO provides the ability to sculpt radiation doses to complex shaped tumor regions while limiting dose to normal organs [6.7]. Compared to conventional IMRT techniques, TOMO may provide sharper dose gradients around the target, which will lead to superior sparing of surrounding normal structures and possibly less radiation-related side effects [8.9.10]. However, the potential benefit of TOMO over IMRT is still being investigated [11.12].

The aim of this study was to compare the therapeutic response (loco-regional control, disease-free, overall survival), toxicities (acute and long-term toxicities) and dosimetric parameters between IMRT and TOMO in patients with advanced cervical cancer, so as to explore an optimal treatment modality for the disease.

\section{Materials And Methods}

\section{Clinical Materials}

In this study, 310 patients were enrolled (KPS $\geq 70$ ) having International Federation of Gynecology and Obstetrics (FIGO 2009) stage $\triangle \mathrm{B}-\triangle \mathrm{B}$ cervical cancer and who received CCRT from August 2015to March 2018 at the Department of Gynecologic Oncology, Shandong Cancer Hospital. A total of 155 patients were treated with IMRT, while the remaining 155 patients underwent TOMO. Intracavitary brachytherapy and concurrent chemotherapy were performed during external beam irradiation. In the IMRT group, ages 
ranged from 28 to 70 years, with a median age of 53 years. Meanwhile, in TOMO group, ages ranged from 26 to 74 years, with a median age of 51 years. The data of clinicopathologic characteristics of all patients were shown in Table 1.

\section{Chemotherapy}

All patients were treated with concurrent chemotherapy during RT. The chemotherapy consisted of infusion of paclitaxel $\left(135 \mathrm{mg} / \mathrm{m}^{2}\right)$ on $\mathrm{d} 1$ and cisplatin $\left(D D P, 75 \mathrm{mg} / \mathrm{m}^{2}\right)$ on $\mathrm{d} 2$ every 4 weeks.

\section{Radiotherapy}

For IMRT, a 6-MV photon beam with six to nine co-planar beams and CT-based treatment planning (Pinnacle version9.2) was used. The doses were delivered using a linear-accelerator (LINAC) equipped with multi-leaf collimators (MLC). The inverse treatment planning was performed using the ADAC Pinnacle ${ }^{3}$ Treatment Planning System (Philips Radiation Oncology Systems, Milpitas, CA, USA). All plans used dynamic multi-leaf collimators (MLC) to shape the fields.

The tomotherapy plans were calculated on the Tomotherapy Planning Station Hi-Art ${ }^{\circledR}$ Version 4.2.3workstation (Tomotherapy Incorporated, Madison, WI, USA) with a superposition/convolution algorithm. Due to work station limitations, CT contouring and OARs images were drawn in Version 9.2 of the Pinnacle3 planning system and transferred to the TOMO planning system.

All patients underwent initial CT simulation in a supine position with their arms by their sides, using intravenous contrast agents and free breathing. A customized immobilization device was fabricated encompassing the lower abdomen, pelvis, and upper thighs to make the daily setup accurate. Scanning range: the upper boundary was at the upper edge of the lumbar 1 vertebral body and the lower boundary was $5 \mathrm{~cm}$ below the ischium tuberosity, scanning layer thickness $5 \mathrm{~mm}$.

Whole pelvic radiation therapy plan was performed to deliver a dosage of $45 \mathrm{~Gy}$ in 1.8 Gy daily fractions, 5 fractions per week in the center of the planning target volume (PTV). The irradiated volume included the whole uterus, cervix and part of the vagina depending upon the lower extent of tumor, the paracervical, parametrium, uterosacral regions as well as common iliac, external iliac, internal iliac and obturator lymph nodes. For patients with pelvic lymph nodes (PLNs) or para-aortic lymph nodes (PALNs) metastasis, para-aortic field radiotherapy plan was also performed at the same time. The prescribed dose was $60 \mathrm{~Gy}$.

Organs at risk(OARs) include suspended ovary, intestinal pouch, rectum, bladder and bilateral femoral head, in which the ovary is the whole ovary marked with silver clip at the lateral paracolonic sulcus, and the intestinal pouch includes the intestinal canal and its surrounding mesenteric tissue shown by contrast medium. the upper boundary of the rectum is the junction of the rectosigmoid colon, the lower boundary is the anus, and the bladder includes all the bladders in the filling state. Dose limitation of OARs: ovary V5

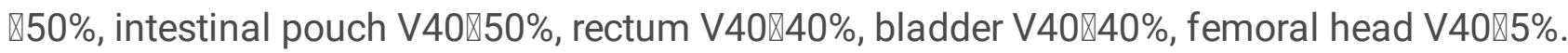


To verify the setup accuracy, we took orthogonal electronic portal images weekly. The gross tumor volume (GTV), CTV and PTV were contoured on the individual axial CT slices of each patient. Normal structures, including the small bowel, rectum, bladder, kidney and pelvic bone marrow were also entered on to the planning CT scan. The rectum was defined from the level of sigmoid flexure to the anus. The small bowel was contoured from the L4-5 interspace to the level of sigmoid flexure.

\section{Dose-volume analysis of treatment plans}

Dose-volume histograms (DVHs) of the PTVs and the critical normal structures were analyzed accordingly. For PTVs, we evaluated the volume, the volume covered by $95 \%$ of the prescription dose (V95), and the minimum doses delivered to 5\% (D5) and 95\% (D95) of the PTV. The critical organs with functional subunits organized in a series were examined. The conformal index $(\mathrm{Cl})$ and the homogeneity index $(\mathrm{HI})$ had been used to evaluate the conformity and uniformity of the plan. The volume received the mean dose for PTV generated from the DVH. The conformal index (CI) for PTV was calculated using the formula $\mathrm{Cl}_{\mathrm{ICRU}}=\mathrm{V}_{\mathrm{TV}} / \mathrm{V}_{\mathrm{PTV}}$, where $\mathrm{V}_{\mathrm{TV}}$ was the ratio of the treated volume enclosed by the prescription isodose surface and $\mathrm{V}_{\text {PTV }}$ was the planning target [13]. The homogeneity index $(\mathrm{HI})$ was defined as $\mathrm{HI}=$ D5/D95, whereD5 and D95 were the minimum doses delivered to $5 \%$ and $95 \%$ of the PTV reported previously.

\section{Intracavitary brachytherapy}

High-dose-rate (HDR) source iridium-192 (Ir-192) was used with a vaginal ovoid applicator (Hanschke applicator set). Post-implantation dosimetry was performed with the Integrated after loading treatment planning system (Shandong, China) and included calculation of dose to Point A bilaterally, pelvic sidewall (Point B, defined as the point $3 \mathrm{~cm}$ from Point $A$ and $5 \mathrm{~cm}$ lateral to midline) bilaterally, the rectal point and bladder point as defined by the International Commission on Radiation Units and Measurements. First, whole pelvic radiotherapy plan was performed to deliver a dosage of $45 \mathrm{~Gy}$. Then intracavitary brachytherapy was administered at doses of $25-30 \mathrm{~Gy}$ in 4 to 5 fractions to after the EBRT course was complete, or in the last week of pelvic EBRT. The planning aim at point A was higher than 85Gy in EQD2. During the treatment of intracavitary brachytherapy, vaginal packing with gauze pushed the bladder and rectum as far as possible to reduce its dose. These treatments were delivered weekly.

\section{Therapeutic effect evaluation}

Therapeutic effect was assessed by clinical examination, ultrasound, CT scans or/and PET-CT scans after 2-3 months of treatment. According to the Response Evaluation Criteria in Solid Tumors, therapeutic response was classified as complete response (CR), partial response (PR), stable disease (SD) and progressive disease (PD).

\section{Toxicity assessment}


The acute and chronic toxicity from radiotherapy were evaluated with the radiation therapy oncology group (RTOG) Criteria in therapy process, after therapy and during follow-up. In patients of grade 4 hematologic or non-hematologic toxicity, radiation therapy was halted until toxicity resolved to at least grade 3.

\section{Follow up}

After treatment completion, patients were followed up at 3months intervals for the first 2 years, and then at 6 months intervals for the following 3 years, and annually thereafter. At each visit, a physical and pelvic examination, blood counts, clinical chemistry, and chest x-rays were performed. Scans of the abdomen and pelvic region were conducted by ultrasound, CT and/or PET-CT when considered clinically indicated. Suspected patients of persistent or recurrent disease were confirmed by biopsy whenever possible. Overall survival (OS) and progression-free survival (PFS) were calculated from the date of diagnosis. Surviving patients were censored on the date of last follow-up. We confirmed the cause of death by telephone, correspondence or medical record review.

\section{Statistical analysis}

The OS and PFS curves were estimated with the Kaplan-Meier method and compared using the log-rank test. Clinical characteristics of patients, toxicities, local control, survival rates and dosimetric parameter were compared using chi-square test between the two groups. Statistical significance was defined at a level of $P<0.05$. All analyses were performed using SPSS version 17.0 (SPSS Inc., Chicago, IL).

\section{Results}

The data on clinicopathologic characteristics of all patients are shown in Table 1 and are similar between the 2 groups. A total of 310 patients were included in the present study. There was no statistical difference in age, FIGO stage, histologic type, tumor size, tumor grade, pathologic morphology type, ovarian conserving between the 2 groups $(P=0.924,0.352,0.954,0.36,0.11,0.123$, and 0.212 , respectively). Pelvic lymph node metastases (PLNM) had occurred in 60 of 310 patients. Among them, there were 18 patients with para-aortic lymph node metastasis(PALNM). And a total of 33 patients (21.3\%) were treated with IMRT and 27 (17.4\%) patients underwent TOMO. There was no statistical difference between the 2 groups $(P=0.388)$.

DVH statistics for OAR and dosimetric parameters were described in Table2. In comparing TOMO with IMRT, better $\mathrm{Cl}(0.82 \pm 0.0327$ vs. $0.75 \pm 0.064, \mathrm{P}=0.006)$ and $\mathrm{HI}(1.03 \pm 0.006$ vs. $1.09 \pm 0.076, \mathrm{p} \nabla 0.0001)$ were observed by TOMO planning. Figures $1 \mathrm{a}$ and $\mathrm{b}$ showed the isodose curves of cross section, sagittal section, and coronary section of a representative patient treated by TOMO or IMRT, which indicates $95 \%$ isodose curve including the PTV. TOMO provided better critical organ sparing than IMRT in the mean dose, and lower bladder V40 ( $p=0.029)$, V20 ( $p=0.001)$, femoral head V40 ( $p=0.014)$, and lower rectum V40 ( $p=0.035)$, V20 ( $p=0.005)$ were observed in the planning by TOMO compared to IMRT for patients with advanced cervical cancer. TOMO demonstrated a superior ability to protect the ovary (Dmax: 4.61Gy 
versus 5.81Gy, $P=0.026$; Dmean: $2.99 \mathrm{~Gy}$ versus 3.97Gy, $P=0.017)$. And femoral head V20 ( $\mathrm{p}=0.066)$, exhibited a tendency toward more favorable values in TOMO than IMRT. There were no statistically significant differences in small bowel V20 $(p=0.251)$, V40 $(p=0.575)$ or bone marrow protection V20 $(p=0.917), V 40(p=0.53)$ between the IMRT plan and the TOMO plan. But it gave significantly better values for Dmax parameters for bone marrow and small bowel with a statistically significant level $(P=0.004$, and 0.002 , respectively). In this study, only 17 patients underwent ovarian suspension. Two patients (33.33\%) of ovarian function was preserved in the IMRT group and 5 (45.5\%) of 11 patients in the TOMO group. There was no statistical difference for ovarian function between the 2 groups $(P=0.627)$.

here was no statistical difference inbetween the 2 groups

Up to November 2019, the median time of follow-up was 32 months (5-53 months) in the IMRT group and 28 months (5-48 months) in the TOMO group. Six (3.87\%) of 155 patients were lost from the IMRT group and $2(1.29 \%)$ of 155 patients from the TOMO group, resulting in the follow-up rates of $96.1 \%$ and $98.7 \%$ $(P=0.175)$, respectively. No significant difference was observed in $C R, P R$, and total response rate (CR+ PR) between TOMO group and IMRT group (CR:94.84\% vs. 95.48\%, P= 0.791; PR:1.9\% vs. 2.5\%, P= 0.709; CR+ PR:96.77\% vs. $98.06 \%, P=0.902$ ). There was no statistical difference for 1- and 3-year OS between the TOMO and IMRT groups ( $94.7 \%$ vs $94.8 \%, \mathrm{P}=0.544 \llbracket 81.5 \%$ vs $84.7 \%, \mathrm{P}=0.413)$. A plot of the survival curves is shown in Figure 2. No obvious difference was found in 1- and3-year PFS rates between 2 groups (1-year: $89.5 \%$ vs $87 \%, \mathrm{P}=0.904$; 3 -year: $80.6 \%$ vs $82 \%, \mathrm{P}=0.708$ ). But for the PLNM patients, there was no statistical difference for 1- and 3-year OS between the TOMO and IMRT groups ( $88.7 \%$ vs $90.9 \%, \mathrm{P}=$ $0.956 \otimes 74.3 \%$ vs $68.9 \%, P=0.882)$. A plot of the survival curves is shown in Figure 3 . No obvious difference was found in 1- and3-year PFS rates between 2 groups with the PLNM patients (1-year: 81.5\% vs $78.8 \%, P=0.843 ; 3$-year: $73.5 \%$ vs $66.2 \%, P=0.956)$. The 1 -and 3 -year OS were $77.8 \%$ and $48.7 \%$ in patient with positive para-aortic lymph nodes, respectively. And the 1-and 3-year PFS were $66.7 \%$ and $52.4 \%$ in patient with PALNM, respectively.

\section{Discussion}

Concurrent chemoradiation is considered to be the standard treatment for patients with locally advanced cervical cancer. Recently, conformal radiotherapy techniques such as 3D-CRT and IMRT have been increasingly used with encouraging results in terms of reduced toxicity owing to relative sparing of normal tissues. Compare with traditional intensity modulated radiotherapy, we found that TOMO can provide patients with cervical cancer with better conformal target coverage, more homogeneous target dose distribution and better bladder and rectum sparing.

Tomotherapy was a 360-degree beam projection degree of freedom in radiotherapy, and the number of sub-fields irradiated by a single dose was more than 20,000. Adding more beams would lead to improved conformality without affecting the value of the objective function [14]. The $\mathrm{Cl}$ was usually larger than 1 , indicating that a portion of the prescription dose was provided outside the PTV. The greater the Cl, the less was the dose conformity to the PTV [12]. The greater HI indicated higher heterogeneity in the PTV 
[15]. Our results showed that compared with intensity modulated radiotherapy, TOMO produced an obvious improvement in the dose conformity $(0.82 \pm 0.0327$ vs. $0.75 \pm 0.064, \mathrm{P}=0.006)$ and homogeneity $(1.03 \pm 0.006$ vs. $1.09 \pm 0.076, p \otimes 0.001)$. Several previous studies have reported that TOMO is superior to IMRT in dose conformity $(0.894 \pm 0.006$ vs. $0.855 \pm 0.008, P=0.000)$ and homogeneity $(1.082 \pm 0.006$ vs. $1.106 \pm 0.006, P=0.023)$ in patients with early cervical cancer and other head and neck cancers [16.17.18].

The ultimate goal of radiotherapy is to improve the dose control rate of the tumor target and reduce the dose of normal tissue as much as possible. Since dose conformity represents the congruence between iso-dose curves and tumor contours [20], better conformity indicates potential superior tumor target coverage and OAR protection. In this study, TOMO provided better critical organ sparing than IMRT in terms of average dose. Compared with IMRT, TOMO observed lower bladder V40 ( $p=0.029)$, bladder V20 $(p=0.001)$, and lower rectum V40 $(p=0.035), \mathrm{V} 20(p=0.005)$ in the planning for patients with advanced cervical cancer. The femoral head V20 $(p=0.066)$, showed a tendency toward more favorable values in TOMO than IMRT. Guo et al [16] reported that a few OARs and dosimetric parameters, including bladder, ovary sparing, rectum, and femoral head(Pख0.001), exhibited more favorable values in TOMO than IMRT for patients with early cervical cancer. This is consistent with several previous studies [20.21.22.23.24] which have indicated that TOMO outperforms IMRT on dose conformity in pelvic tumors. And the volume of low-dose irradiation of the intestinal, pelvis and normal tissues decreased in patients with TOMO.

It is well known that pelvic radiotherapy can cause a variety of complications, including small intestinal obstruction, radiation cystitis, urinary incontinence, fistula and pelvic fractures [26]. Consistent with these dosimetric data, reduced toxicities in the practice of treating cancer in the pelvic cavity were expected with the use of TOMO. Some retrospective studies compared the incidence of toxicities in IMRT and TOMO, and presented positive results of TOMO [24.26]. In this study, seventeen (11\%) patients in the IMRT group and $5(3.22 \%)$ in the TOMO group experienced grade $3 / 4$ acute proctitis. Four (2.58\%) patients in the IMRT group and $3(1.94 \%)$ in the TOMO group experienced grade $3 / 4$ acute cystitis. The acute radiation toxicity of proctitis, and cystitis was significantly lower in TOMO group than IMRT group $(P=0.034$, and 0.049 , respectively). This is in concordance with the studies of Chang [27] and Yao et al [24] which indicated that protection of the bladder and rectum is a significant advantage of TOMO when compared with IMRT. TOMO can decrease the risk of radiation-induced toxicity in patients with pelvic radiotherapy.

Ovarian transposition is mainly suitable for young patients with cervical cancer who need pelvic radiotherapy. Before pelvic radiotherapy, the arteries and veins of ovary were dissected, ovarian blood supply was preserved, and ovaries were moved outside the irradiation field to avoid the effect of radiotherapy on ovarian function. The success of ovarian function preservation after radiotherapy is related to many factors, such as the dose of ovary during radiotherapy, the age of patients, the location of ovarian displacement, whether there is the concurrent chemotherapy and so on. And the dose of ovary during radiotherapy is the most important factor that directly affects ovarian endocrine function. Therefore, postoperative radiotherapy planning is required to minimize the dose of radiation to the ovaries. TOMO can produce more complex radiation fields because of changing the design of multi-leaf collimator. It can not only ensure the uniformity and conformity of dose in target area, but also avoid 
OARs that need to be protected in the ray path. At the same time, its radiation contamination is smaller, so that the OARs can be accurately avoided [27]. For the displaced ovaries, the target area and ovarian dose can be better considered, and the possibility of making concessions in target range and conformity is less. And each precise image guidance can minimize the placement error, better ensure the accurate implementation of the plan, achieve precise treatment, and add a layer of precise protection for ovarian function. Ovary is a parallel organ. The damage of ovarian cells during radiotherapy is directly related to the ovarian function. In this subject, TOMO demonstrated a superior ability to protect the ovary (Dmax: 4.61Gy versus $5.81 \mathrm{~Gy}, \mathrm{P}=0.026$; Dmean: $2.99 \mathrm{~Gy}$ versus 3.97Gy, $\mathrm{P}=0.017$ ). Guo et al [16] reported that TOMO provided better ovarian organ sparing than IMRT in the mean dose\and the difference was statistically significant $(P \otimes 0.001)$. Therefore, TOMO radiotherapy is recommended for young patients who need pelvic radiotherapy. But, only two patients $(33.33 \% ; 2 / 6)$ of ovarian function was preserved in the IMRT group and $5(45.5 \%)$ of 11 patients in the TOMO group. There was no statistical difference for ovarian function between the 2 groups $(P=0.627)$. Although there was no significant difference between the two groups, the ratio of TOMO group was higher than that of IMRT group. This could be related to the small sample size of this study. Further prospective randomized multicenter studies are needed to confirm the benefits of TOMO.

There were few published studies exploring tumor control comparing IMRT with TOMO for locally advanced cervical carcinoma patients. The present study demonstrated that similar tumor response rate between the two groups. No significant difference was observed in CR, PR, and total response rate (CR+ PR) between TOMO group and IMRT group (CR:94.84\% vs. 95.48\%, P= 0.791; PR:1.9\% vs. 2.5\%, P= 0.709; CR+ PR:96.77\% vs. $98.06 \%, P=0.902$ ). There was no statistical difference for 1- and 3-year OS between the TOMO and IMRT groups ( $94.7 \%$ vs $94.8 \%, P=0.544 \otimes 81.5 \%$ vs $84.7 \%, P=0.413$ ). No obvious difference was found in 1- and3-year PFS rates between 2 groups (1-year: $89.5 \%$ vs $87 \%, P=0.904$; 3 year: $80.6 \%$ vs $82 \%, P=0.708)$. Wang et al [28] reported outcomes of patients with stages IB1-IVA cervical cancer treated with definitive IMRT. The 3-year OS, PFS rates were $83.0 \%$, and $75.0 \%$, respectively. Chang et al [26] included 15 patients with stage IB1-IVA cervical cancer in their study, all received pelvic irradiation delivered by TOMO. The 3-year OS was 93\%, 3-year PFS rate was $80 \%$. These results indicate that TOMO is feasible for locally advanced cervical carcinoma patients. But, these also show that TOMO will not greatly improve the recurrence rate and mortality of patients, shorten the recurrence time of patients, so that the treatment effect of patients can be effectively guaranteed, which is worthy of further clinical research.

\section{Conclusions}

Our results show that TOMO and IMRT are comparable in mean dose, dose conformity, dose homogeneity, and protection of the ovary. TOMO provided better critical organ sparing than IMRT in the lower bladder, and lower rectum were observed in the planning. The acute and chronic toxicities were

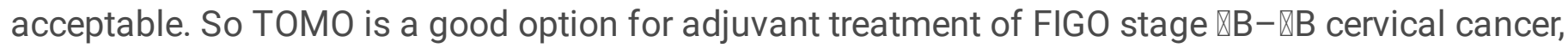
especially to young patients. Further prospective randomized multicenter studies are needed to confirm the benefits of TOMO. 


\section{Abbreviations}

IMRT: Intensity-modulated radiation therapy

TOMO: Tomotherapy

CCRT: Concurrent chemoradiation

FIGO: International Federation of Gynecology and Obstetrics

EBRT: External beam radiation therapy

BT: Bachytherapy

DDP: Cisplatin

LINAC: Linear-accelerator

MLC: Multi-leaf collimators

PTV: Planning target volume

PLNs: Pelvic lymph nodes

PALNs: Para-aortic lymph nodes

OARs: Organs at risk

GTV: Gross tumor volume

CTV: Clinical tumor volume

DVHs: Dose-volume histograms

Cl: Conformal index

HI: Homogeneity index

CR: Complete response,

PR: Partial response

SD: Stable disease

PD: Progressive disease.

OS: Overall survival 
PFS: Progression-free survival

PLNM: Pelvic lymph node metastases

PALNM: Para-aortic lymph node metastasis

GI: Gastrointestinal

GU: Genitourinary

\section{Declarations}

\section{Availability of data and materials}

The datasets used and/or analyzed during the current study are available from the corresponding author on reasonable request.

Acknowledgements Not applicable.

Funding This study was supported by Key Technology Research and Development Program of Shandong (CN). [No.2018GSF118237].

Author information Yueju Yin and Dandan Wang contributed equally to this work.

\section{Author contributions.}

Yueju Yin recorded and analyzed the experimental raw data, was a major contributor in writing the manuscript. Dandan Wang collected the clinical date and revised the manuscript. Quancai Chen and Jue Jia were contributors to recorded the experimental raw data. Shuai Feng and Hao Yu designed the experiment and revised the manuscript. Jian Zhu designed the experiment, and analyzed DVH date. Dapeng Li designed the experiment, checked the experimental raw data and was a major contributor revised the manuscript.

\section{Corresponding author}

Correspondence to Dapeng Li and Jian Zhu.

\section{Ethics declarations}

\section{Ethics approval and consent to participate}

Institutional review board approval from the Shandong Tumor Hospital and Institute was obtained before undertaking this study. 
Not applicable.

\section{Competing interests}

The authors have no potential conflicts of interest to report.

\section{References}

1. Bhatla, N. Aoki, D. Sharma, D. N. Sankaranarayanan, R. Cancer of the cervix uteri. Int J Gynecol Obstet 2018; 143 (2): 22-36.

2. Chen W, Zheng R, Baade PD, Zhang S, Zeng H, Bray F, et al. Cancer statistics in China,2015. CA Cancer J Clin. 2016; 66: 115-32.

3. Dutta S, Nguyen NP, Vock J, Kerr C, Godinez J, Bose S, et al. Image-guided radiotherapy and brachytherapy for cervical cancer. Front Oncol. 2015; 5:64.

4. Harkenrider MM, Alite F, Silva SR, Small W Jr. Image-based brachytherapy for the treatment of cervical cancer. Int J Radiat Oncol Biol Phys. 2015; 92:921-934.

5. Mackie TR, Holmes T, Swerdloff S, Reckwerdt P, Deasy JO, Yang J, et al. Tomotherapy: A new concept for the delivery of dynamic conformal radiotherapy. Med Phys 1993; 20:1709-19.

6. Mackie TR, Kapatoes J, Ruchala K, Lu W, Wu C, Olivera G, et al. Image guidance for precision conformal radiotherapy. Int J Radiat Oncol Biol Phys 2003; 56:89-105.

7. Balog J, Mackie TR, Pearson D, Hui S, Paliwal B, Jeraj R. Benchmarking beam alignment for a clinical helical tomotherapy device. Med Phys. 2003; 30:1118-27.

8. Grigorov G, Kron T, Wong E, Chen J, Sollazzo J, Rodrigues G. Optimization of helical tomotherapy treatment plans for prostate cancer. Phys Med Biol. 2003; 48:1933-43.

9. van Vulpen M, Field C, Raaijmakers $\mathrm{CP}$, Parliament MB, Terhaard $\mathrm{CH}$, Mackenzie MA, et al. Comparing step-and-shoot IMRT with dynamic helical tomotherapy IMRT plans for head-and-neck cancer. Int J Radiat Oncol Biol Phys. 2005; 62:1535-9.

10. Chen YJ, Liu A, Han C, Tsai PT, Schultheiss TE, Pezner RD, et al. Helical Tomotherapy for radiotherapy in esophageal cancer: a preferred plan with better conformal target coverage and more homogeneous dose distribution. Med Dosim. 2007; 32:166-71.

11. Lian J, Mackenzie M, Joseph K, Pervez N, Dundas G, Urtasun R, et al. Assessment of extended-field radiotherapy for stage IIIC endometrial cancer using three-dimensional conformal radiotherapy, intensity-modulated radiotherapy, and helical tomotherapy. Int J Radiat Oncol Biol Phys. 2008;70(3):935-943.

12. Hsieh $\mathrm{CH}$, Wei MC, Lee HY, Hsiao SM, Chen CA, Wang LY, et al. Whole pelvic helical tomotherapy for locally advanced cervical cancer: technical implementation of IMRT with helical tomotherapy. Radiat Oncol. 2009; 4:62.

13. Forrest J, Presutti J, Davidson M, Hamilton P, Kiss A, Thomas G. A dosimetric planning study comparing intensity-modulated radiotherapy with four-field conformal pelvic radiotherapy for the 
definitive treatment of cervical carcinoma. Clin Oncol (R Coll Radiol). 2012;24(4): e63-e70.

14. Wang X, Zhang X, Dong L, Liu H, Gillin M, Ahamad A, et al. Effectiveness of noncoplanar IMRT planning using a parallelized multiresolution beam angle optimization method for paranasal sinus carcinoma. Int J Radiat Oncol Biol Phys 2005,63:594-601.

15. Chen ZY, Ma YB, Sheng XG, Zhang XL, Xue L, Song QQ, et al. [Intensity modulated radiation therapy for patients with gynecological malignancies after hysterectomy and chemotherapy/radiotherapy]. Zhonghua Zhong Liu Za Zhi, 2007, 29(4):305-308.

16. Guo MF, Liu XF, Liu L, Wang D, et al. [A dosimetric study of helical tomotherapy in radiotherapy after ovarian-conserving radical surgery for young patients with early-stage cervical cancer]. Journal of Chongqing Medical University, 2019, 044(001):39-42.

17. Wiezorek T, Brachwitz T, Georg D, Blank E, Fotina I, Habl G, et al. Rotational IMRT techniques compared to fixed gantry IMRT and tomotherapy: multi-institutional planning study for head-andneck cases. Radiat Oncol. 2011; 6:20.

18. Clemente S, Wu B, Sanguineti G, Fusco V, Ricchetti F Wong J, et al. Smart Arc-based volumetric modulated arc therapy for oropharyngeal cancer: a dosimetric comparison with both intensitymodulated radiation therapy and helical tomotherapy. Int J Radiat Oncol Biol Phys. 2011;80(4):12481255.

19. Feuvret L, Noël G, Mazeron JJ, Bey P. Conformity index: a review. Int J Radiat Oncol Biol Phys. 2006;64(2):333-342.

20. Yang R, Xu S, Jiang W, Wang J, Xie C. Dosimetric comparison of postoperative whole pelvic radiotherapy for endometrial cancer using three-dimensional conformal radiotherapy, intensitymodulated radiotherapy, and helical tomotherapy. Acta Oncol. 2010;49(2):230-236.

21. Engels B, De Ridder M, Tournel K, Sermeus A, Coninck P, Verellen D, et al. Preoperative helical tomotherapy and megavoltage computed tomography for rectal cancer: impact on the irradiated volume of small bowel. Int J Radiat Oncol Biol Phys. 2009;74(5):1476-1480.

22. Tournel K, De Ridder M, Engels B, Bijdekerke P, Yves Fierens Y, Duchateau M, et al. Assessment of intrafractional movement and internal motion in radiotherapy of rectal cancer using megavoltage computed tomography. Int J Radiat Oncol Biol Phys. 2008;71(3):934-939.

23. Gattaneo GM, Dell'oca I, Broggi S, Fiorino C, Perna L, Pasetti M, et al. Treatment planning comparison between conformal radiotherapy and helical tomotherapy in the case of locally advanced - staged NSCLC. Radiother Oncol, 2008, 88(3): 310-318.

24. Yao B, Wang SH, Wang YD, Liu QZ, Lu N, et al. Acute and late toxicities and efficacy of helical tomotherapy and concurrent chemotherapy in the treatment of locally advanced cervical cancer. Oncol Prog, 2016, 14(6): 544-547.

25. Rose BS, Aydogan B, Liang Y, Mete Yeginer M, Hasselle M D, Dandekar V, et al. Normal tissue complication probability modeling of acute hematologic toxicity in cervical cancer patients treated with chemoradiotherapy. Int J Radiat Oncol Biol Phys. 2011;79(3):800-807. 
26. Chang AJ, Richardson S, Grigsby PW, Schwarz JK. Split-field helical tomotherapy with or without chemotherapy for definitive treatment of cervical cancer. Int J Radiat Oncol Biol Phys. 2012;82(1):263-269.

27. Lin M A, Radiotherapy D O. Clinical Application of Tomotherapy System. China Medical Devices, 2014.

28. Wang W, Zhang F, Hu K, Hou X. Image-guided, intensity-modulated radiation therapy in definitive radiotherapy for 1433 patients with cervical cancer. Gynecol Oncol. 2018;151(3):444-448.

\section{Tables}




\begin{tabular}{|c|c|c|c|}
\hline Characteristics & IMRT group $(n=155)$ & TOMO group $(n=155)$ & P-Value \\
\hline Age (years, median [range]) & 28-70(53) & $26-74(51)$ & 0.924 \\
\hline FIGOstage & & & 0.352 \\
\hline$\bigotimes \mathrm{B}$ & 46 & 57 & \\
\hline$\triangle \mathrm{A}$ & 3 & 4 & \\
\hline$\bigotimes \mathrm{B}$ & 106 & 94 & \\
\hline Histologic type & & & 0.954 \\
\hline Squamous carcinoma & 146 & 147 & \\
\hline Adenocarcinoma & 6 & 5 & \\
\hline Other type & 3 & 3 & \\
\hline Tumor size, cm & & & 0.36 \\
\hline$\leq 4 \mathrm{~cm}$ & 64 & 72 & \\
\hline$\otimes 4 \mathrm{~cm}$ & 91 & 83 & \\
\hline Tumor grade & & & 0.11 \\
\hline G1 & 48 & 54 & \\
\hline G2 & 50 & 61 & \\
\hline G3 & 57 & 40 & \\
\hline Ovarian conserving & & & 0.212 \\
\hline No & 149 & 144 & \\
\hline Yes & 6 & 11 & \\
\hline Pathologic morphology type & & & 0.123 \\
\hline Exophytic & 135 & 125 & \\
\hline Endophytic type & 20 & 30 & \\
\hline
\end{tabular}




\begin{tabular}{|c|c|c|c|c|}
\hline \multirow[t]{2}{*}{ Parameter } & & \multicolumn{3}{|l|}{ Mean \pm SD } \\
\hline & & TOMO Groups & IMRT Groups & P-value \\
\hline & D5\% & $52.56 \pm 0.28$ & $54.82 \pm 0.22$ & 0.001 \\
\hline & D95\% & $50.82 \pm 0.31$ & $50.27 \pm 0.27$ & 0.001 \\
\hline & $\mathrm{HI}$ & $1.03 \pm 0.006$ & $1.09 \pm 0.076$ & 0.001 \\
\hline & $\mathrm{Cl}$ & $0.82 \pm 0.033$ & $0.75 \pm 0.064$ & 0.006 \\
\hline \multirow[t]{5}{*}{ Bladder } & V40 (\%) & $27.31 \pm 7.16$ & $34.13 \pm 7.97$ & 0.029 \\
\hline & V20 (\%) & $66.34 \pm 8.82$ & $80.36 \pm 8.16$ & 0.001 \\
\hline & Dmean (Gy) & $29.28 \pm 3.01$ & $33.07 \pm 3.21$ & 0.01 \\
\hline & Dmin (Gy) & $10.55 \pm 1.43$ & $9.93 \pm 2.40$ & 0.47 \\
\hline & Dmax (Gy) & $53.34 \pm 0.88$ & $56.35 \pm 3.22$ & 0.007 \\
\hline \multirow[t]{5}{*}{ Femoral head-L } & V40 (\%) & $0.57 \pm 0.49$ & $1.16 \pm 0.57$ & 0.014 \\
\hline & V20 (\%) & $45.46 \pm 4.89$ & $38.64 \pm 10.57$ & 0.066 \\
\hline & Dmean (Gy) & $21.46 \pm 1.07$ & $19.84 \pm 2.40$ & 0.054 \\
\hline & Dmin (Gy) & $15.31 \pm 0.93$ & $10.39 \pm 2.18$ & 0.001 \\
\hline & Dmax (Gy) & $42.89 \pm 2.84$ & $42.73 \pm 5.88$ & 0.935 \\
\hline \multirow[t]{5}{*}{ Femoral head-R } & V40 (\%) & $0.50 \pm 0.55$ & $1.43 \pm 1.01$ & 0.014 \\
\hline & V20 (\%) & $46.35 \pm 5.65$ & $41.36 \pm 9.08$ & 0.138 \\
\hline & Dmean (Gy) & $21.60 \pm 0.61$ & $20.03 \pm 2.26$ & 0.037 \\
\hline & Dmin (Gy) & $15.11 \pm 0.81$ & $8.10 \pm 2.42$ & 0.001 \\
\hline & Dmax (Gy) & $42.67 \pm 2.27$ & $44.28 \pm 4.94$ & 0.337 \\
\hline \multirow[t]{5}{*}{ Rectum } & V40 (\%) & $22.82 \pm 6.53$ & $29.18 \pm 6.66$ & 0.035 \\
\hline & V20 (\%) & $63.41 \pm 11.94$ & $79.36 \pm 12.01$ & 0.005 \\
\hline & Dmean (Gy) & $28.00 \pm 3.20$ & $32.35 \pm 2.88$ & 0.003 \\
\hline & Dmin (Gy) & $12.08 \pm 1.52$ & $12.39 \pm 2.79$ & 0.751 \\
\hline & Dmax (Gy) & $52.41 \pm 1.03$ & $55.24 \pm 3.29$ & 0.007 \\
\hline \multirow[t]{2}{*}{ Small bowel } & V40 (\%) & $21.01 \pm 9.09$ & $23.18 \pm 8.75$ & 0.575 \\
\hline & V20 (\%) & $66.25 \pm 10.00$ & $61.55 \pm 8.63$ & 0.251 \\
\hline
\end{tabular}




\begin{tabular}{|lllll|}
\hline & Dmean (Gy) & $26.74 \pm 3.57$ & $26.48 \pm 3.46$ & 0.865 \\
\hline Dmin (Gy) & $1.90 \pm 0.40$ & $1.79 \pm 0.66$ & 0.627 \\
\hline Bone marrow & Dmax (Gy) & $28.00 \pm 3.20$ & $28.00 \pm 3.20$ & 0.002 \\
\hline & V40 (\%) & $26.81 \pm 6.08$ & $24.82 \pm 8.33$ & 0.53 \\
\hline V20 (\%) & $74.42 \pm 8.05$ & $74.82 \pm 9.36$ & 0.917 \\
\hline Dmean (Gy) & $29.09 \pm 2.73$ & $32.37 \pm 3.47$ & 0.619 \\
\hline Ovary-L & Dmin (Gy) & $3.60 \pm 3.88$ & $4.75 \pm 7.12$ & 0.641 \\
\hline & Dmax (Gy) & $53.71 \pm 0.98$ & $56.89 \pm 3.04$ & 0.004 \\
\hline Dmean (Gy) & $2.99 \pm 0.65$ & $3.97 \pm 1.05$ & 0.017 \\
\hline Ovary-R & Dmin (Gy) & $2.04 \pm 0.55$ & $2.71 \pm 0.55$ & 0.01 \\
\hline & Dmax (Gy) & $4.61 \pm 1.26$ & $5.81 \pm 1.07$ & 0.026 \\
\hline & Dmean (Gy) & $2.98 \pm 0.59$ & $3.84 \pm 0.73$ & 0.007 \\
\hline & Dmin (Gy) & $1.83 \pm 0.55$ & $2.47 \pm 0.43$ & 0.007 \\
\hline & Dmax (Gy) & $4.53 \pm 0.88$ & $5.87 \pm 1.37$ & 0.013 \\
\hline
\end{tabular}

D95: the percentage of the prescribed dose covering $95 \%$ volume of PTV; D5: the percentage of the prescribed dose covering 5\% volume of PTVXIMRT: intensity modulated radiation therapy; TOMO: tomotherapy; $\vee x$ : the percentage of organ receiving more or equal to $x$ Gy $₫ S D$ : standard deviation; 
Table3 Crude incidence of acute toxicity in both groups according to RTOG/EORTC acute radiation morbidity criteria in patients with cervical cancer

\begin{tabular}{|c|c|c|c|}
\hline Grade & IMRT group $(n=155)$ & TOMO group $(n=155)$ & P-Value \\
\hline Leukopenia & & & 0.025 \\
\hline 0 & 15 & 34 & \\
\hline 1 & 14 & 16 & \\
\hline 2 & 55 & 45 & \\
\hline 3 & 47 & 46 & \\
\hline 4 & 24 & 14 & \\
\hline Cystitis & & & 0.049 \\
\hline 0 & 57 & 78 & \\
\hline 1 & 66 & 60 & \\
\hline 2 & 27 & 14 & \\
\hline 3 & 4 & 3 & \\
\hline 4 & 1 & 0 & \\
\hline Enteritis & & & 0.082 \\
\hline 0 & 54 & 62 & \\
\hline 1 & 67 & 77 & \\
\hline 2 & 30 & 14 & \\
\hline 3 & 3 & 2 & \\
\hline 4 & 1 & 0 & \\
\hline Proctitis & & & 0.034 \\
\hline 0 & 53 & 71 & \\
\hline 1 & 67 & 64 & \\
\hline 2 & 18 & 15 & \\
\hline 3 & 16 & 5 & \\
\hline 4 & 1 & 0 & \\
\hline Dermatitis & & & 0.616 \\
\hline 0 & 56 & 61 & \\
\hline 1 & 72 & 73 & \\
\hline
\end{tabular}




\begin{tabular}{|c|c|c|}
\hline 2 & 27 & 21 \\
\hline 3 & 3 & 0 \\
\hline 4 & 0 & 0 \\
\hline
\end{tabular}

Table4 Chronic toxicities in both groups according to RTOG/EORTC acute radiation morbidity criteria in patients with cervical cancer

\begin{tabular}{|llll|}
\hline Grade & IMRT group $(\mathrm{n}=155)$ & TOMO group $(\mathrm{n}=155)$ & P-Value \\
\hline Cystitis & & 59 & 0.048 \\
\hline 0 & 46 & 72 & \\
1 & 64 & 18 & \\
\hline 2 & 34 & 6 & 0.032 \\
\hline 3 & 9 & 0 & \\
\hline 4 & 2 & & \\
\hline Enterocolitis & & 55 & \\
\hline 0 & 40 & 70 & \\
\hline 1 & 60 & 24 & \\
\hline 2 & 45 & 5 & \\
\hline 4 & 8 & 1 & \\
\hline
\end{tabular}

\section{Figures}



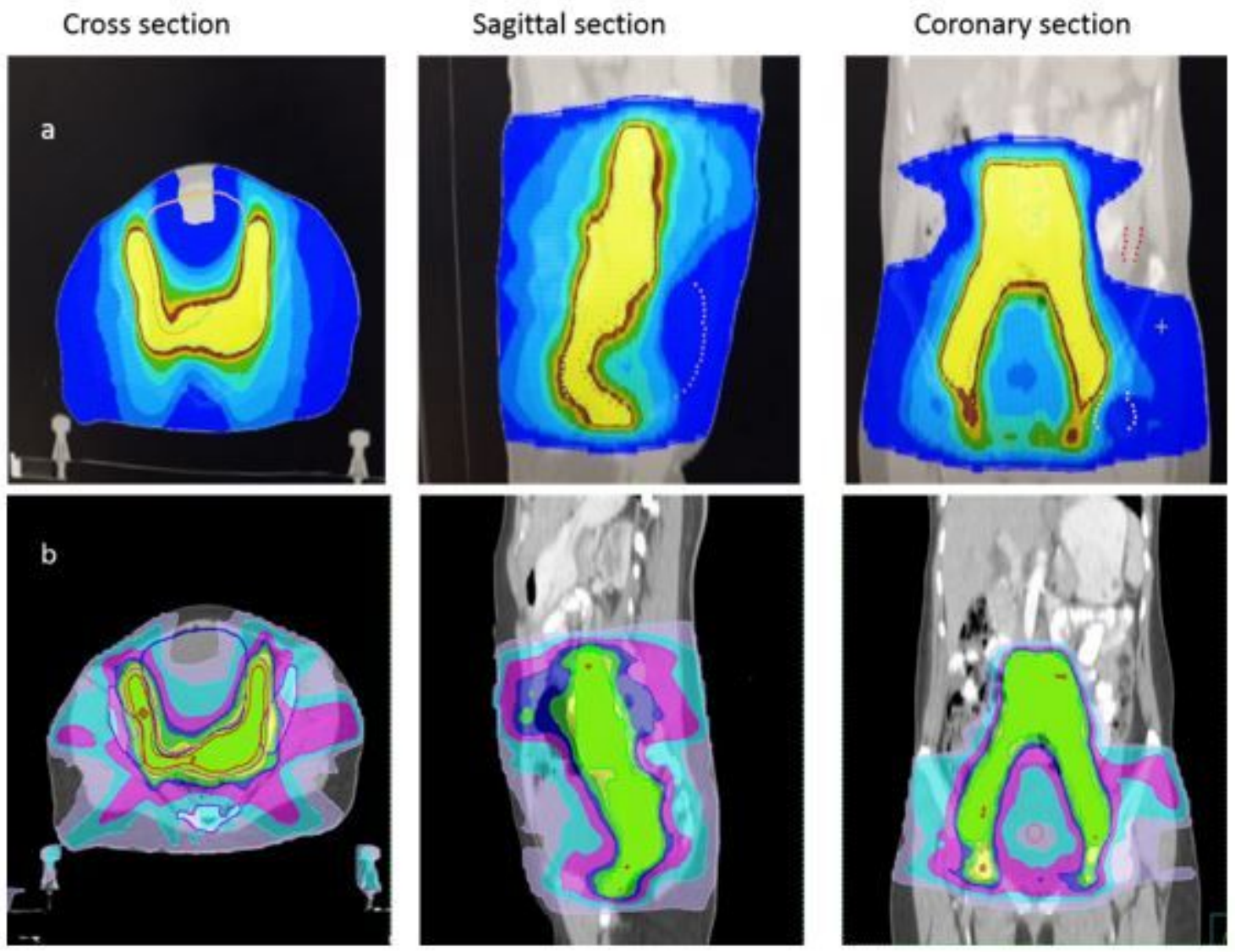

\section{Figure 1}

Isodose curves of a cross section, sagittal section and coronary section of one representative patient for TOMO (a) and one patient for IMRT (b).
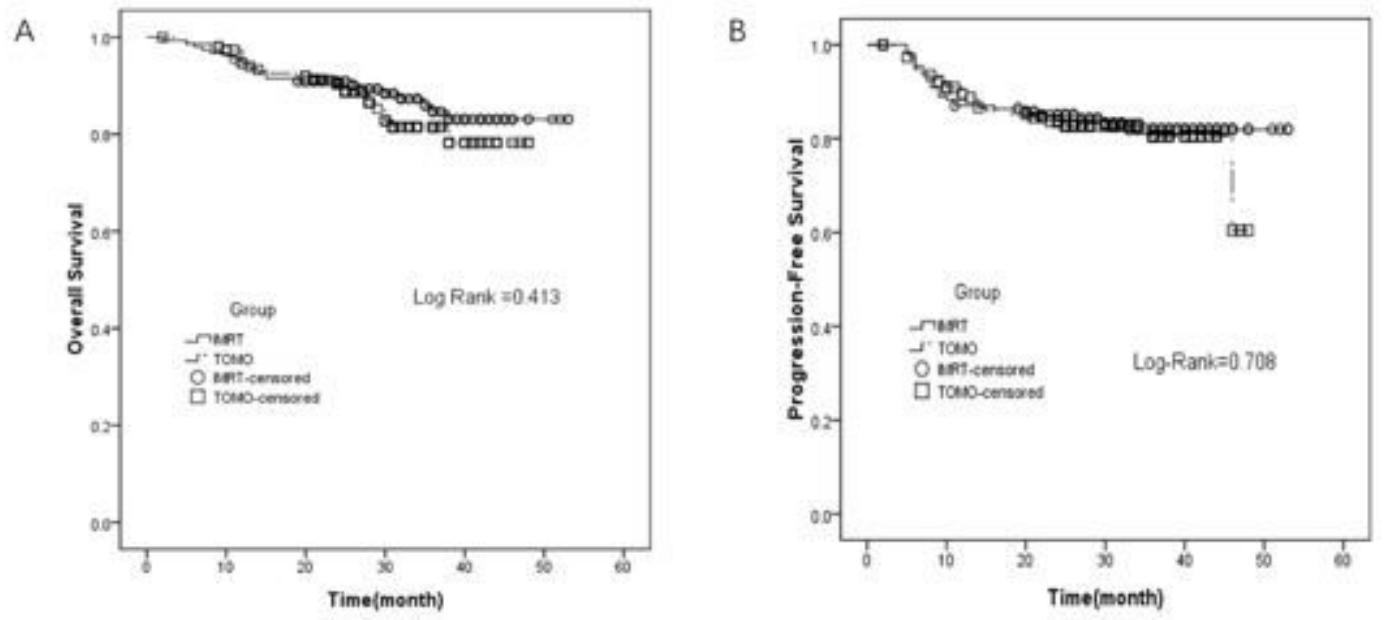

Figure 2 
Survival curve (Kaplan-Meier method): OS and PFS for patients with advanced cervical cancer: (A) OS, (B) PFS.

A

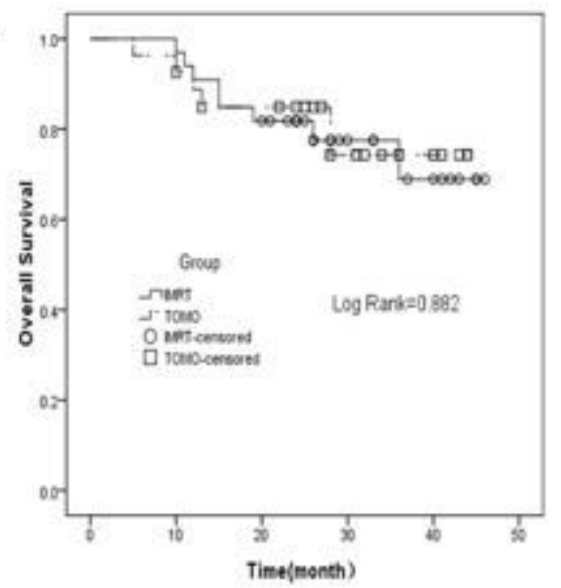

B

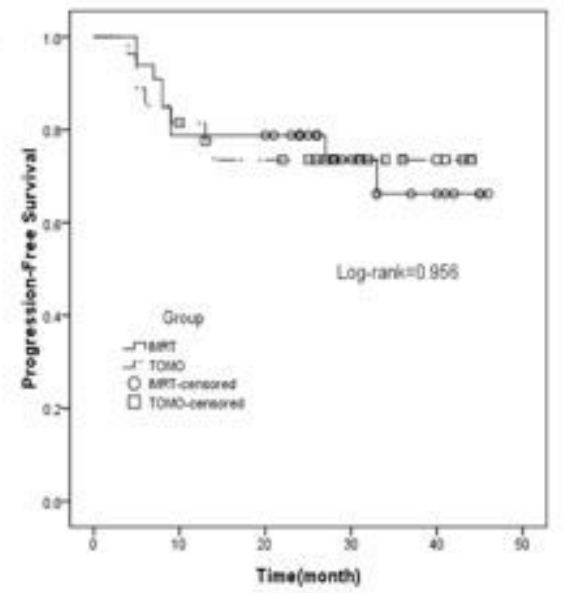

\section{Figure 3}

Survival curve (Kaplan-Meier method): OS and PFS for patients with the PLNM: (A) OS, (B) PFS. 